UVX 2012, 01007 (2013)

DOI: $10.1051 /$ uvx/201301007

(C) Owned by the authors, published by EDP Sciences, 2013

\title{
Sources $X$ : du multi-keV-ns au multi-MeV-ps
}

\author{
M. Primout, L. Jacquet, D. Babonneau, X. Davoine, \\ et A. Compant La Fontaine
}

\author{
CEA/DAMIDIF, 91297 Arpajon Cedex, France
}

\begin{abstract}
Résumé. Plusieurs concepts étudiés pour la réalisation des sources $\mathrm{X}$ du domaine multi-keV/ns au domaine multi-MeV/ps sont abordés. Ces sources de rayonnement sont développées dans le but de radiographier les cibles sur le Laser Mégajoule (LMJ). Pour valider ces concepts et pour prédimensionner ces sources de radiographie, de nombreuses campagnes expérimentales ont été menées sur des installations comme ALISE, GEKKO XII, OMEGA, OMEGA-EP et LIL.
\end{abstract}

\section{INTRODUCTION}

Dans ce qui suit, les principes de fonctionnement de sources $\mathrm{X}$ dans un vaste domaine énergétique allant du multi-keV au multi-MeV et dans un domaine temporel de la picoseconde à la nanoseconde sont évoqués. Les besoins radiographiques sur le LMJ imposent des critères qui sont liés à la nature de la cible à radiographier et des matériaux à traverser. Les caractéristiques de l'émission X des photons en découlent directement (essentiellement l'énergie, la monochromaticité et la durée d'émission). Cela entraine des régimes d'interaction laser-matière très différents.

Dans la gamme inférieure à $10 \mathrm{keV}$, en régime ns et pour les énergies radiographiques disponibles sur le LMJ, il est possible de créer des plasmas «thermiques » où l'émission de type $\mathrm{He}_{\alpha}$ domine, avec des matériaux de numéro atomique faible, du titane au germanium, par exemple. Au delà de $10 \mathrm{keV}$, il devient plus difficile d'obtenir des températures électroniques qui permettent une émission $\mathrm{He}_{\alpha}$ efficace avec des matériaux de numéro atomique plus élevé sauf à augmenter l'éclairement laser de plusieurs ordres de grandeur. Dans cette gamme d'éclairement laser $\left(\gg 10^{16} \mathrm{~W} / \mathrm{cm}^{-2}\right)$, d'autres mécanismes d'interaction laser-matière interviennent comme l'accélération d'électrons suprathermiques par le champ laser qui conduisent à d'autres types de sources X par Bremmstrahlung, $\mathrm{K}_{\alpha}$ ou betatron.

\section{LES SOURCES X MULTI-KeV DANS LE DOMAINE D'IRRADIATION kJ/ns}

Ces sources $\mathrm{X}$ multi-keV/ns sont utilisées pour la radiographie d'objets denses dans le contexte de la fusion par confinement inertiel (FCI). Différents concepts pour la réalisation de sources dans ce domaine d'irradiation laser $\mathrm{kJ} / \mathrm{ns}$, ont été étudiés et développés par le CEA en collaboration avec plusieurs laboratoires depuis 2002 sur différentes installations.

Dans ce domaine, l'émission X multi-keV de ces sources, dites «thermiques », est du type $\mathrm{He}_{\alpha}$ car elle repose sur la création d'un plasma chaud et ionisé jusqu'à l'état héliumoïde. Dans cette gamme d'énergie, à l'exception d'éléments de Z plus élevé comme le Xénon (couche L), l'émission multi-keV correspond à des transitions radiatives impliquant le niveau de couche $\mathrm{K}$.

Pour rendre plus efficace ces sources métalliques, plusieurs voies ont été explorées théoriquement et expérimentalement depuis le début des années 2000. La première d'entre elles consiste à se servir de cibles solides initialement sous-denses, constituées d'aérogels à dopant métallique [1,2] ou d'oxydes

This is an Open Access article distributed under the terms of the Creative Commons Attribution License 2.0, which permits unrestricted use, distribution, and reproduction in any medium, provided the original work is properly cited. 


\section{Web of Conferences}
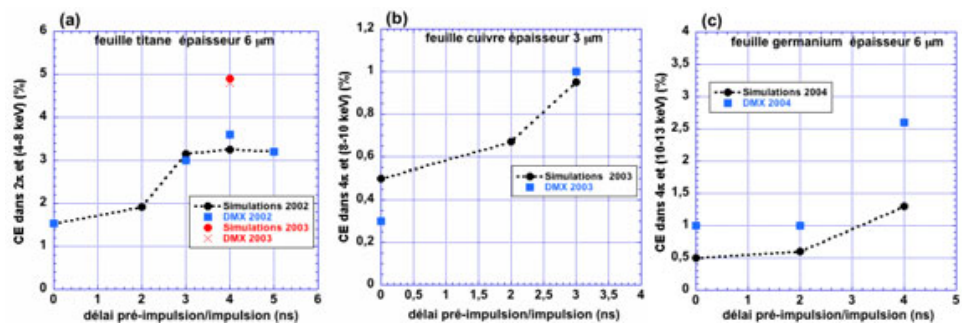

Figure 1. Rendements de conversion en fonction du délai pré-impulsion/impulsion principale pour (a) des feuilles de titane, (b) des feuilles de cuivre, (c) des feuilles de germanium.

métalliques [3]. Une seconde voie est d'utiliser une partie, si possible faible, de l'énergie laser disponible pour créer un plasma sous-dense. Cela a conduit au concept de feuille mince pré-explosée [4,5]. Enfin, une troisième voie a consisté à utiliser le confinement hydrodynamique radial du plasma dans une cible de géométrie cylindrique qui a conduit au concept de cavité tapissée de métal [6-9].

\subsection{Les feuilles minces pré-explosées}

Afin d'augmenter les taux de conversion X (CE), on cherche à créer, à partir d'une feuille solide, un plasma sous-critique, l'énergie d'une préimpulsion laser servant à créer une large couronne sous-dense avant l'impulsion laser principale. Un délai optimisé (quelques ns) sépare par la préimpulsion laser de l'impulsion principale, permettant une production accrue de rayonnement X multi-keV. Dans cette configuration, le CE dépend de plusieurs paramètres : l'épaisseur de la feuille, les caractéristiques de la pré-impulsion et de l'impulsion principale (éclairement, durée, énergie) et le délai entre les deux impulsions. Une analyse, basée sur un grand nombre de simulations 1D et un traitement statistique des résultats obtenus, permet de déterminer de façon optimale l'épaisseur des feuilles et le délai entre les impulsions [4].

Des campagnes, utilisant ces configurations de cibles, ont eu lieu sur OMEGA $(2002,2003,2004)$ afin de valider expérimentalement ce concept de feuilles pré-explosées. Les épaisseurs des feuilles valaient $6 \mu \mathrm{m}$ pour le Ti, $3 \mu \mathrm{m}$ pour le $\mathrm{Cu}, 6 \mu \mathrm{m}$ pour le $\mathrm{Ge}$. Les énergies déposées par la pré-impulsion variaient de $\sim 1.3 \mathrm{~kJ}$ à $\sim 2 \mathrm{~kJ}$ suivant les tirs et celles de l'impulsion principale de $\sim 3.3 \mathrm{~kJ}$ à $\sim 7 \mathrm{~kJ}$. La Fig. 1 montre l'augmentation des rendements en fonction du retard entre la préimpulsion et l'impulsion principale.

\subsection{Les cavités tapissées de métal}

Les feuilles minces pré-explosées ont montré l'intérêt de créer un plasma chaud et sous-dense pour obtenir de forts taux de conversion X multi-keV. On peut cependant penser que leurs performances sont limitées par la détente du plasma en géométrie ouverte. Dans des géométries en cavité, l'effet de confinement hydrodynamique permet à la fois d'augmenter la densité et la température électronique du plasma émissif et de maintenir sur une plus longue durée les conditions favorables à la production d'énergie multi-keV. Les sources en cavité sont des cylindres de plastique intérieurement tapissés par un fin dépôt métallique (liner), de quelques $\mu$ m d'épaisseur. La Fig. 2 montre une cavité tapissée de germanium et une demi-cavité tapissée de titane.

La première campagne CEA d'étude de sources en cavité a eu lieu sur OMEGA en 2005 où six tirs ont été réalisés, toutes les cavités ayant des liners de Ti et étant irradiées des 2 côtés. Les énergies délivrées étaient comprises entre $\sim 14.5 \mathrm{~kJ}$ et $\sim 17 \mathrm{~kJ}$. Pour la deuxième campagne CEA en 2007, cinq tirs ont lieu sur OMEGA. Une demi-cavité tapissée de Ti, deux cavités tapissées de Ti et deux demicavités tapissées de Ge ont été expérimentées. Les énergies délivrées étaient comprises entre $\sim 5.8 \mathrm{~kJ}$ et 


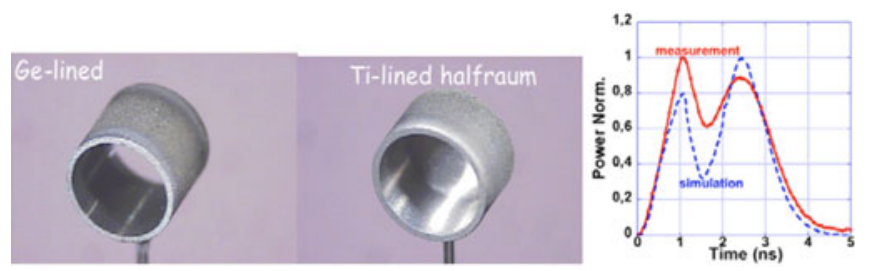

Figure 2. Sources $X$ en cavité : tapissée de germanium en irradiation bilatérale (gauche), demi-cavité tapissée de titane en schéma d'irradiation unilatérale (milieu) et impulsions $\mathrm{X}$ typiques normalisées (droite).

$\sim 6.4 \mathrm{~kJ}$ pour les cibles irradiées d'un seul côté. Une énergie de $\sim 13 \mathrm{~kJ}$ a été délivrée à la cible irradiée de façon bilatérale [10].

Une autre campagne a eu lieu sur OMEGA en 2009 avec des cavités ou demi-cavités tapissées de titane, cuivre ou germanium, en irradiation uni ou bilatérales et des énergies entre 6 et $17 \mathrm{~kJ}$. Un certain nombre de désaccords sont observés entre les simulations avec le code FCI2 et les mesures expérimentales, plus importants dans le cas du titane que dans le cas du germanium. Les valeurs maximales de CE mesurés sont voisines de $8 \%$ pour le Ti et $0,5 \%$ pour le Ge. La Fig. 2 (à droite) montre une comparaison des impulsions $\mathrm{X}$ calculées et simulées entre 4 et $6 \mathrm{keV}$ (en valeurs normalisées) : un bon accord qualitatif est observé qui montre une bonne restitution de l'hydrodynamique de la cavité mais un facteur de l'ordre de 2 subsiste entre les énergies totales calculées et simulées.

\subsection{Les sources hybrides (aérogel/feuille/cylindre)}

Récemment nous avons testé un nouveau concept de cibles hybrides : elles sont constituées d'un cylindre de plastique rempli d'aérogel sous dense et obturé par une feuille de titane. Le but de ce concept était (1) de transporter l'énergie du laser incident, la convertir en une onde thermique supersonique pour aller chauffer la feuille de titane placée à l'autre extrémité du cylindre et (2) de réaliser un double confinement : radial et longitudinal pour empêcher la feuille de titane de trop se détendre pendant la phase de chauffage. Le but est d'obtenir une augmentation du rendement de conversion X en optimisant la longueur et le diamètre du cylindre ainsi que la densité de l'aérogel. Concrètement ces cibles ont été réalisées à partir de cylindres de plastique de 0,5 à $1 \mathrm{~mm}$ de longueur, de diamètre entre 1 et $2 \mathrm{~mm}$, remplis d'aérogel de silice à des densités de 2 et $5 \mathrm{mg} / \mathrm{cm}^{3}$ et obturés par une feuille mince $(3 \mu \mathrm{m})$ ou épaisse $(20 \mu \mathrm{m})$ de titane.

\section{SOURCE $\mathrm{K}_{\alpha}$ ET BETATRON POUR LA RADIOGRAPHIE X SUR LE Imj}

Nous présentons les principes de fonctionnement de deux types de sources $\mathrm{X}$ ultra brèves, les sources $\mathrm{K}_{\alpha}$ et betatron, qui pourraient être envisagées pour la radiographie X sur le LMJ avec le laser PETAL. Ces sources ont l'avantage d'avoir une durée inférieure à la ps, permettant de réaliser une radiographie avec une très bonne résolution temporelle et une petite taille $(1$ à $10 \mu \mathrm{m})$ pour radiographier un objet avec une bonne résolution spatiale. Pour simuler ces sources au CEA nous développons et utilisons les codes CALDER et CALDER-CIRC qui sont des codes PIC (Particles-In-Cell) servant à modéliser l'interaction laser-plasma.

\subsection{Source $\mathbf{K}_{\alpha}$}

Le rayonnement $\mathrm{K}_{\alpha}$ est produit lorsqu' un laser ultra intense interagit avec une cible solide (Fig. 3). Le laser ionise la cible et génère des électrons suprathermiques de plusieurs dizaines de keV. Ces électrons 


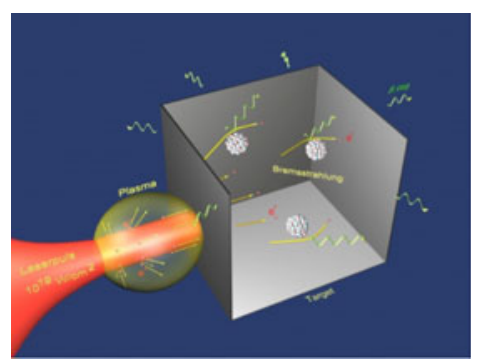

Figure 3. Principe d'une source $\mathrm{K}_{\alpha}$. Le laser interagit avec une cible solide et produit un plasma en face avant. L'interaction laser-plasma accélère ensuite des électrons jusqu'à plusieurs dizaines de keV. Ces électrons se propagent dans la cible et les collisions avec les atomes de la cible peuvent provoquer l'émission de rayonnement $\mathrm{K}_{\alpha}$.

Tableau 1. Énergies de transition $\mathrm{K} \alpha_{1}$ et $\mathrm{L} \alpha_{1}$ pour différents éléments.

\begin{tabular}{|l|c|l|c|c|c|}
\hline & Aluminium & Cuivre & Argent & Or & Uranium \\
\hline $\mathrm{K}_{\alpha 1}$ & $1487 \mathrm{eV}$ & $8048 \mathrm{eV}$ & $22,2 \mathrm{keV}$ & $68,8 \mathrm{keV}$ & $98,4 \mathrm{keV}$ \\
\hline $\mathrm{L}_{\alpha 1}$ & & $930 \mathrm{eV}$ & $2985 \mathrm{eV}$ & $9713 \mathrm{eV}$ & $13,6 \mathrm{keV}$ \\
\hline
\end{tabular}

énergétiques peuvent alors ioniser à leur tour la matière en profondeur. Lorsqu'un électron sur une couche externe comble un trou créé en couche interne $\mathrm{K}$, un rayonnement de fluorescence $\mathrm{X}$ est alors émis. Ce type de source a une émission isotrope, monochromatique avec une énergie de 5 à plusieurs dizaines de keV selon les atomes constituant la cible. Sa durée sera de l'ordre de la picoseconde avec un laser comme PETAL. La taille de ces sources $\mathrm{K}_{\alpha}$ est typiquement de l'ordre de la centaine de microns lorsque le laser interagit avec une grande cible car même si la tache laser est plus petite, les électrons suprathermiques se répartissent sur une zone plus large. Cette taille est un paramètre important car elle limite la résolution spatiale de la radiographie : il n'est pas possible de voir des détails plus petits que la taille de source. Pour la réduire, il a été proposé d'utiliser un fil ayant une section de l'ordre de 5-10 microns comme cible. La taille de source, limitée par l'extension de la cible, peut ainsi être réduite à 5-10 microns [11,12].

Les électrons rapides accélérés par le laser ps possèdent généralement un spectre en énergie étendu, depuis l'énergie d'agitation thermique (de l'ordre de l'eV) jusqu'à des énergies relativistes (dizaine de $\mathrm{MeV}$ voire plus). Seuls les électrons de la partie haute-énergie du spectre peuvent engendrer du rayonnement $\mathrm{K}_{\alpha}$. En effet, un électron ne pourra exciter la raie $\mathrm{K}_{\alpha}$ que si l'énergie cinétique $\mathrm{E}$ qu'il possède est supérieure à l'énergie d'ionisation $\mathrm{I}_{\mathrm{K}}$ de la couche $\mathrm{K}$. Par exemple, pour le cuivre, $\mathrm{I}_{\mathrm{K}}=8979 \mathrm{eV}$. La lacune ainsi produite dans la couche K est rapidement comblée par les électrons des couches supérieures. Notamment, un électron de la couche $L\left(2 p_{1 / 2}\right.$ ou $\left.2 p_{3 / 2}\right)$ peut transiter vers cette lacune et émettre un photon dont l'énergie est égale à la différence entre le niveau de départ $E_{\mathrm{L}}$ et celui d'arrivée $\mathrm{E}_{\mathrm{K}}$. Le tableau 1 montre les énergies auxquelles les photons $\mathrm{K}_{\alpha}$ sont émis pour différents éléments.

Pour se rendre compte de l'efficacité de l'ionisation en couche K, la section efficace $\sigma_{K}$ en fonction de E est représentée dans la Fig. 4 pour divers éléments. La section efficace est bien évidemment nulle en-dessous d' $\mathrm{I}_{\mathrm{K}}$, et relativement constante au-dessus de $2 \mathrm{I}_{\mathrm{K}}$ dans la gamme d'énergie considérée.

Ainsi, dans le cas du cuivre, l'ionisation en couche interne est efficace pour un électron d'énergie cinétique supérieure à $20 \mathrm{keV}$ environ. Pour l'instant, CALDER et MCNP permettent d'obtenir une simulation en deux étapes du rayonnement $\mathrm{K}_{\alpha}$. 

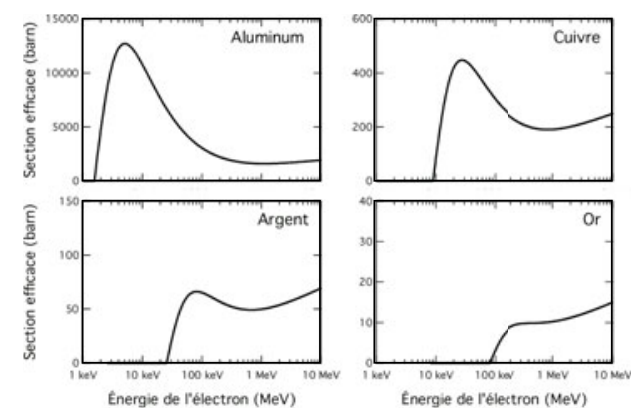

Figure 4. Section efficace $\sigma_{\mathrm{K}}$ d'ionisation en couche $\mathrm{K}$ par impact électronique en fonction de l'énergie de l'électron incident, pour différents matériaux

\subsection{Rayonnement betatron}

Le rayonnement betatron est engendré dans les accélérateurs d'électrons par sillage laser. L'interaction d'un laser ultra intense avec un jet de gaz (de quelques $\mathrm{mm}$ à quelques $\mathrm{cm}$ ) permet de créer une onde plasma dans le sillage du laser. Les champs électriques associés à cette onde accélèrent très fortement les électrons et les font osciller transversalement. Ces électrons émettent alors un rayonnement de type synchrotron, appelé rayonnement betatron. Ces sources X sont caractérisées par un spectre large (probablement de quelques $\mathrm{keV}$ à plusieurs dizaines de $\mathrm{keV}$ voire plus avec PETAL), une forte collimation (de l'ordre de la dizaine de mrad), une durée qui devrait être inférieure à la ps avec PETAL (de l'ordre de la dizaine voire centaine de fs), et une taille de source de l'ordre du micron.

L'avantage des sources betatron sur les sources $\mathrm{K}_{\alpha}$ est leur très bonne collimation et leur petite taille de source. Leur bonne collimation assure habituellement d'avoir un nombre de photons sur cible bien plus important, d'où une radiographie de meilleure qualité. Leur petite taille de source permet d'avoir naturellement une très bonne résolution spatiale de l'objet à radiographier (de l'ordre de la taille de la source). Plusieurs preuves de principe ont déjà montré expérimentalement que ces sources permettaient d'observer des détails assez fins grâce à la technique de radiographie par contraste de phase [13].

Cependant, comme expliqué ci-dessous, il reste à prouver qu'il est possible de créer de telles sources avec PETAL, car les lasers habituellement utilisés pour engendrer ces sources betatron ont des durées d'impulsion beaucoup plus courtes.

\subsection{Sources betatron sur PETAL ?}

Pour engendrer efficacement une onde de sillage, la durée de l'impulsion laser doit idéalement être inférieure à la période de l'onde plasma de sillage, qui dépend directement de la densité du plasma. Pour le laser PETAL, la durée optimale serait au maximum de l'ordre de $150 \mathrm{fs}$ à $200 \mathrm{fs}$. A priori ce laser ne semble donc pas adapté pour engendrer efficacement une onde de sillage à moins d'entrer dans un régime d'auto-modulation où le laser est modulé par l'onde plasma de faible amplitude qu'il va d'abord créer. Grâce à cette modulation, l'impulsion laser acquiert des caractéristiques suffisantes pour engendrer efficacement une onde de sillage et accélérer des électrons. Cependant, ce régime d'automodulation n'a principalement été étudié que pour des lasers de plus faible puissance que PETAL. La physique est suffisamment bien comprise pour qu' on s' attende à obtenir des électrons et du rayonnement betatron avec le laser PETAL, cependant il reste difficile d'estimer avec précision les caractéristiques du rayonnement qui sera émis. 

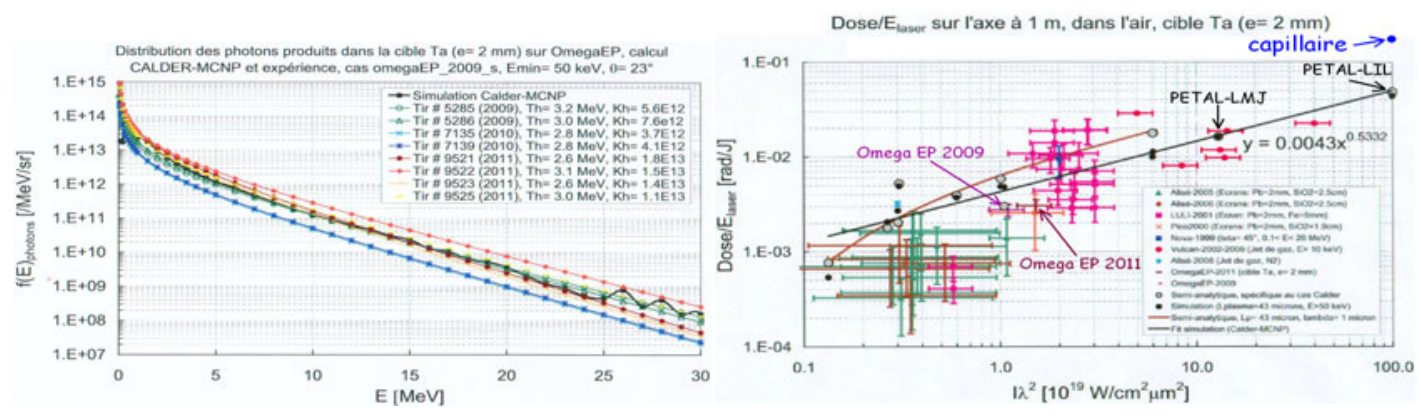

Figure 5. Gauche : fonction de distribution de l'énergie des photons sur OmegaEP pour les expériences de 2009, 2010 et 2011. Mesures expérimentales et simulation numérique CALDER-MCNP. Droite : dose sur l'axe à $1 \mathrm{~m}$ dans l'air.

\section{SOURCES X MULTI-MEV PRODUITES PAR LASERS À ULTRA-HAUTE INTENSITÉ}

L'étude des sources X multi-MeV entre dans le cadre des études de faisabilité d'une source de radiographie éclair produite par interaction laser-plasma avec un laser à ultra-haute intensité (UHI). Pendant l'interaction d'un laser à haute intensité PW/ps avec un plasma, une grande quantité d'électrons relativistes sont accélérés. Si ces électrons interagissent avec une cible solide de Z élevé placée derrière la zone d'interaction, des photons sont créés par Bremsstrahlung.

Les expériences effectuées sur un grand nombre d'installations laser sur le laser P102 du CEA/LV [14], sur NOVA[15], sur VULCAN[16,17], au LOA et au LULI [18] ont produit une grande quantité de rayonnement $\mathrm{X}$ dans la gamme multi-MeV et permis de radiographier des objets divers [19]. Les études expérimentales et théoriques menées à la DIF dans ce domaine ont permis de préciser les caractéristiques des sources $\mathrm{X}$ multi-MeV obtenues en fonction de celles du laser et des conditions d'interaction, passant de quelques centaines de TW dans les années 80 aux lasers PW actuels. Si l'on interpose une cible solide de numéro atomique élevé, ces d'électrons multi-MeV relativistes ainsi produits engendrent des photons de haute énergie par conversion Bremsstrahlung, constituant une source $\mathrm{X}$ utilisable pour la radiographie d'objets denses.

\subsection{Résultats sur Omega EP}

Une série d'expériences ont été effectuées sur Omega EP de Rochester de 2008 à 2011 avec des cibles minces d'or de 20 et $100 \mu \mathrm{m}$ d'épaisseur. En 2008, les conditions laser étaient : durée d'impulsion $\Delta t_{\mathrm{FWHM}}=0,6 \mathrm{ps}$, énergie laser sur cible $\mathrm{E}=300 \mathrm{~J}$, tache focale $\phi_{\mathrm{FWHM}}=30 \mu \mathrm{m}$, soit $\mathrm{a}_{0}=5,69$ et $\mathrm{I}_{0}=4,4 \times 10^{19} \mathrm{~W} / \mathrm{cm}^{2}$ avec une injection en polarisation $\mathrm{p}$ à $45^{\circ}$ par rapport au plan de la cible [20]. Cette mise en place a permis de valider les différents diagnostics utilisés et d'étudier la circulation multiple des électrons dans la cible de $20 \mu \mathrm{m}$. Les expériences suivantes de 2009 et 2010 [21,22] ont été effectuées avec la même dimension de tache laser. Par contre l'énergie sur cible a été portée à $\mathrm{E}_{\text {laser }}=1 \mathrm{~kJ}$ et la durée d'impulsion $\Delta \mathrm{t}=8$ et $10 \mathrm{ps}$ respectivement, ce qui donne $\mathrm{a}_{0}=2,76$ et 2,47 et $\mathrm{I}_{0}=1,0510^{19} \mathrm{~W} / \mathrm{cm}^{2}$ et $8,410^{18} \mathrm{~W} / \mathrm{cm}^{2}$ respectivement. En 2011 , l'énergie sur cible a été portée à $\mathrm{E}_{\text {laser }}=1,5 \mathrm{~kJ}$, avec $\Delta \mathrm{t}=9 \mathrm{ps}$, soit $\mathrm{a}_{0}=3,35$ et $\mathrm{I}_{10} 0^{19} \mathrm{~W} / \mathrm{cm}^{2}$. Le spectre expérimental en énergie de photons a été obtenu comme pour les expériences sur ALISE à l'aide des mesures d'activation dans des disques en cuivre et carbone et en prenant pour distribution en énergie des photons, celle correspondant à une distribution en énergie des électrons obtenue par un calcul PIC : $f_{\text {electrons }}(E)=v_{0} E \exp \left(-E / T_{h}\right)$, dont l'intégration angulaire sur la section efficace de Bremsstrahlung donne $: f_{\text {photons }}(E)=K_{h}\left[\left(1-b+T_{h} / E\right) e^{-E / T_{h}}+\left(b-E_{0} / E-T_{h} / E\right) e^{-E_{0} / T_{h}}\right]$. 
Les résultats expérimentaux sont représentés sur la Fig. 5 (gauche). Les résultats de simulation donnés pour les tirs de 2009 sont en bon accord avec les résultats expérimentaux. Le niveau de l'émission photonique est environ 2 ordres de grandeur supérieur à celui obtenu précédemment sur ALISE, comme le rapport des énergies laser de ces deux installations, en accord avec la loi d'échelle : Dose $[\mathrm{rad}] / \mathrm{E}_{\text {laser }}[\mathrm{kJ}] \sim \mathrm{a}_{0}$.

On donne sur la Fig. 5 (droite) les valeurs de dose par unité d'énergie laser en fonction de I $\lambda^{2}$, obtenues par la simulation numérique Calder-MCNP et expérimentalement sur différentes installations laser (avec les barres d'erreurs). La configuration « standard » considérée ici, correspond à l'interaction d'un laser ps en polarisation $\mathrm{p}$ sur un préplasma de longueur caractéristique $\mathrm{L}_{\mathrm{p}}=15 \mu \mathrm{m}$ interagissant avec une cible solide en tantale de $2 \mathrm{~mm}$ d'épaisseur, excepté pour les cas particuliers indiqués. L'expression semi analytique est représentée pour $L_{p}=43 \mu \mathrm{m}$. On donne aussi sur cette figure les résultats de la simulation dans la configuration PETAL sur la LIL et dans celle de PETAL sur le LMJ.

\subsection{Perspectives pour PETAL}

Une première étude porte sur la caractérisation des sources $\mathrm{X}$ multi-MeV et de la dose de la future installation du laser pétawatt PETAL dans le contexte antérieur d'implantation sur la LIL ( $\lambda=1 \mu \mathrm{m}$, $\mathrm{I}=10^{21} \mathrm{~W} / \mathrm{cm}^{2}, \mathrm{a}_{0}=27, \mathrm{E}_{\text {laser }}=840 \mathrm{~J}, \Delta t_{F W H M}^{\text {laser }}=0,7 \mathrm{ps}, \mathrm{P}=1,2 \mathrm{PW}, \varphi_{F W H L M}^{\text {laser }}=10 \mu \mathrm{m}$ avec un rapport de Strehl de 0,25 inclus. Notons que dans la perspective du couplage de PETAL au LMJ, l'intensité laser chute environ d'un ordre de grandeur par rapport au contexte antérieur de couplage à la LIL. Par suite de l'augmentation de la taille de la tache focale, la dose sur l'axe, obtenue par simulation Calder-MCNP sur cible solide avec un préplasma de longueur caractéristique $\mathrm{L}_{\mathrm{p}}=14 \mu \mathrm{m}$, chute de 35 rads (cas PETAL-LIL) à 17 rads (cas PETAL-LMJ), selon la loi d'échelle du paragraphe précédent.

\section{CONCLUSION}

Nous avons testé une grande variété de sources $\mathrm{X}$ dans un large domaine énergétique et temporel : dans le domaine multi-keV/ns, des bons rendements de conversion ont été obtenus. Les simulations doivent encore progresser, en particulier dans le domaine des modèles HETL couplés au code hydrodynamique, pour mieux rendre compte de l'ensemble des mesures effectuées. Dans le domaine des lasers ultra intenses et ultra courts les outils sont en place pour continuer les études menées sur l'installation OMEGA-EP et les extrapoler vers PETAL. Le calcul complet de l'émission $\mathrm{K}_{\alpha}$ devrait permettre d'optimiser des cibles dans le domaine d'énergie souhaité. La démonstration de la possibilité de réaliser des sources betatron est encore à démontrer mais là aussi les progrès de la simulation et la compréhension des phénomènes physiques acquise au cours de ces dernières années devraient permettre d'envisager un dimensionnement de ces sources sur PETAL à brève échéance.

\section{Références}

[1] K. B. Fournier et al., Phys. Rev. Lett. 92, 165005 (2004)

[2] K. B. Fournier et al., Phys. Plasmas. 16, 052703 (2009)

[3] A. E. Gash et al., Chem. Mater. 15, 3268 (2003)

[4] M. Primout, J. X-Ray Sci. Technol. 13, 23 (2005)

[5] D. Babonneau et al., Phys. Plasmas 15, 092702 (2008)

[6] D. Babonneau et al., Bull. Am. Phys. Soc. 47 (9) 312 (2002)

[7] F. Girard et al., Phys. Plasmas 16, 052704 (2009)

[8] F. Girard et al., Phys. Plasmas 18, 079901 (2011)

[9] L. Jacquet et al., soumis à Physics of Plasmas

[10] M. Primout et al., J. Phys. : Conf. Ser. 112, 042051 (2008) 


\section{Web of Conferences}

[11] H.-S. Park et al., POP 13056309 (2006)

[12] H.-S. Park et al., POP 15072705 (2008)

[13] S. Fourmaux et al., Opt. Letters 362426 (2011)

[14] G. Malka and J. L. Miquel, Phys. Rev. Lett. 77, 75 (1996)

[15] M. D. Perry et al., Rev. Sci. Instrum. 70, 265 (1999)

[16] P. A. Norreys et al. Phys. Plasmas 6, 2150 (1999)

[17] R. D. Edwards et al., Appl. Phys. Lett. 80, 2129 (2002)

[18] E. Lefebvre et al., Nucl. Fusion 43, 629 (2003)

[19] Y. Glinec et al., Phys. Rev. Lett. 94, 025003 (2005)

[20] A. Pukhov, Report Progress in Physics 66, 47 (2003)

[21] C. Courtois et al., Phys. Plasmas 18, 023101 (2011)

[22] A. Compant La Fontaine et al., Phys. Plasmas, 19, 023104 (2012) 